\title{
GA $_{3}$ e Paclobutrazol no florescimento e na produção de frutos em duas cultivares de morangueiro
}

\author{
Jaime Duarte Filho ${ }^{1}$; Luís Eduardo C. Antunes ${ }^{2}$; Joaquim G. de Pádua ${ }^{1}$ \\ ${ }^{1}$ Epamig, Fazenda Experimental de Caldas, C. Postal 33, 37780-000 Caldas-MG; E-mail: duartefilho@epamigcaldas.gov.br; ${ }^{2}$ Embrapa \\ Clima Temperado, Br 392 Km 78, 96001-970 Pelotas-RS
}

\section{RESUMO}

Neste trabalho estudou-se o efeito dos fitorreguladores $\mathrm{Ga}_{3}$ e Paclobutrazol no florescimento e produção de frutos de morangueiro (Fragaria x ananassa Duch.). O experimento foi conduzido em Caldas (MG), na EPAMIG. O delineamento experimental adotado foi o de blocos casualizados, com quatro repetições, num esquema fatorial $2 \times 4$, onde as variáveis dependentes foram duas cultivares ('Oso Grande' e 'Seascape') e os tratamentos controle (água); $\mathrm{GA}_{3}$ a $40 \mathrm{mg} \mathrm{L}^{-1} \mathrm{em}$ duas aplicações (aos 45 e 65 dias após o transplante); paclobutrazol (produto comercial Cultar $10 \%$ ) a $100 \mathrm{mg} \mathrm{L}^{-1}$ aos 75 dias após o transplante das mudas; combinação de $\mathrm{GA}_{3}+$ paclobutrazol aplicados aos 45; 65 e aos 75 dias, respectivamente, após o transplante. Os tratamentos foram aplicados via foliar em ambas as cultivares. A resposta à aplicação dos fitorreguladores $\mathrm{GA}_{3}$ e paclobutrazol em morangueiro depende da cultivar. A cv. Seascape, de dia neutro, respondeu melhor à aplicação de $\mathrm{GA}_{3}$ que a 'Oso Grande', de dia curto; Por outro lado, o paclobutrazol, quando aplicado via foliar, aos 75 dias após o transplante, reduziu o florescimento do morangueiro o que mostra a necessidade de mais pesquisas sobre dosagens e épocas de aplicação do paclobutrazol nesta cultura.

Palavras-chave: Fragaria x ananassa Duch., fitorreguladores, fenologia.

\begin{abstract}
GA3 and Paclobutrazol on the flowering and yield of strawberry

The effect of bioregulators $\mathrm{GA}_{3}$ and paclobutrazol was evaluated on flowering and yield of strawberry cultivars Oso Grande and Seascape. The experiment was carried out in Caldas, Minas Gerais State, Brazil. The treatments consisted of control (no application of bioregulators); $\mathrm{GA}_{3}$ at $40 \mathrm{mg} \mathrm{L}^{-1}$, in two applications (45 and 65 days after transplanting date); paclobutrazol (commercial product Cultar, $10 \%$ ) at $100 \mathrm{mg} \mathrm{L}^{-1}, 75$ days after transplanting date; combination of $\mathrm{GA}_{3}+$ paclobutrazol, applied 45; 65 and 75 days, respectively, after transplanting date. The experimental design was a randomized complete block, with four replications, in a factorial $2 \mathrm{x} 4$. The response to the bioregulators $\mathrm{GA}_{3}$ and Paclobutrazol was cultivar dependent. 'Seascape', a day neutral cultivar, presented better results in relation to application of $\mathrm{GA}_{3}$ than 'Oso Grande', a shortday cultivar. Paclobutrazol applied 75 days after transplanting date reduced the flowering.
\end{abstract}

Keywords: Fragaria $x$ ananassa Duch., plant growth regulator, phenology.

(Recebido para publicação em 15 de maio de 2003 e aceito em 05 de janeiro de 2004)

$\mathrm{A}$ tualmente, o morangueiro é cultivado em inúmeras regiões do território brasileiro, com destaque para os estados de Minas Gerais (maior produtor nacional), São Paulo e Rio Grande do Sul (Duarte Filho et al., 1999). Além desses três Estados, o morangueiro é ainda cultivado no Paraná, Espírito Santo, Santa Catarina, Brasília, Bahia, entre outros, principalmente em função da maior rentabilidade proporcionada por essa cultura. Este fato tem estimulado sua expansão em diferentes regiões, mesmo sendo uma culturas que exige intenso e meticuloso trabalho durante o ciclo e onerosa operação de colheita e embalagem, devido à fragilidade dos seus frutos. (Lucchesi e Minami 1980).

Entretanto, apesar do incremento da área plantada, a cultura vem apresentando baixa produtividade $(26,1 \mathrm{t} / \mathrm{ha})$, quando comparada à de outros países produtores, como Espanha com produ- tividade média de 30 a 35 t/ha (Roudeillac, 1999). Isto se deve, entre outros fatores, ao baixo nível tecnológico adotado pela maioria dos nossos produtores.

A aplicação de técnicas avançadas, desde a fertilização, passando pela irrigação e pelo uso do plástico, até a utilização de reguladores e inibidores de crescimento que, segundo Castro et al. (1995), podem melhorar quantitativa e qualitativamente a frutificação do morangueiro, é plenamente justificada. Com relação aos reguladores de crescimento, atualmente o ácido giberélico é o mais investigado na cultura do morangueiro, visando incrementar a produção.

Em Valencia, Lopez-Galarza et al. (1989) verificaram notável aumento na produção precoce de morangueiros cv. Douglas com aplicação de ácido giberélico a $40 \mathrm{mg} \mathrm{L}^{-1}$ durante o inverno. Porém, na produção total e tamanho dos frutos foi pequeno o incremento, sem diferir estatisticamente das plantas não tratadas. Nas condições de Piracicaba (SP), Castro et al. (1995), verificaram que aplicações de ácido giberélico a $50 \mathrm{mg}$ $\mathrm{L}^{-1}$ na cv. Campinas, aos 58 e 65 dias após o transplante, aumentaram o número e o peso dos frutos.

Inibidores de crescimento em culturas hortícolas são cada vez mais avaliados em trabalhos de pesquisa, objetivando principalmente a precocidade e o aumento da produtividade. Os produtos pertencentes ao grupo dos inibidores da biossíntese da giberelina, [CCC (Cloreto de 2-cloroetil-trimetil amônio), paclobutrazol e uniconazole], em geral têm proporcionado aumento na produtividade do morangueiro (Ramina et al. 1985; Deyton et al. 1991; Nishizawa, 1993). Entretanto, efeitos adversos como inibição do florescimento e redução da frutificação 


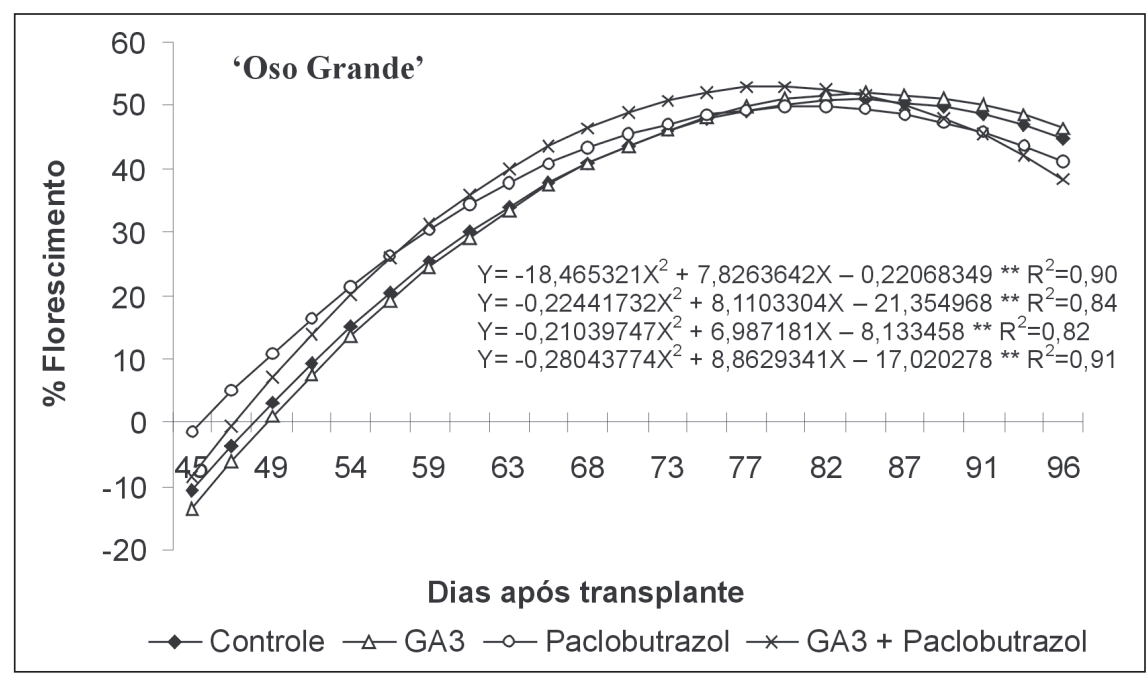

Figura 1. Porcentagem de florescimento, ao longo do período avaliado, do morangueiro cv. Oso Grande, em função dos diferentes fitorreguladores utilizados. Dados transformados em $\operatorname{arcsen} \sqrt{ } \mathrm{x} / 100$. Caldas, EPAMIG, 1999.

são relatados na literatura. Esses efeitos variam em função de fatores como momento da aplicação, cultivar, condições climáticas, entre outros.

O objetivo do trabalho foi avaliar o efeito dos fitorreguladores $\mathrm{GA}_{3} \mathrm{e}$ paclobutrazol no florescimento e na produção total de duas cultivares de morangueiro, uma de dia curto e outra de dia neutro, nas condições do Planalto de Poços de Caldas, em Minas Gerais.

\section{MATERIAL E MÉTODOS}

$\mathrm{O}$ trabalho foi desenvolvido na $\mathrm{Fa}-$ zenda Experimental da EPAMIG (FECD) em Caldas (MG), durante o ano de 1999. O experimento teve início em 24/03/99 com o transplante de mudas uniformes produzidas no local, a partir de matrizes propagadas por cultura de meristema, em canteiros com 1,20 m de largura e $25 \mathrm{~cm}$ de altura, preparados com 30 dias de antecedência, com a incorporação de fertilizantes e corretivos.

O delineamento experimental adotado foi de blocos casualizados, com quatro repetições, no esquema fatorial $2 \mathrm{X} 4$, onde as variáveis dependentes foram cultivar 'Oso Grande' (dia curto) e cultivar 'Seascape' (dia neutro) e os tratamentos controle (água); $\mathrm{GA}_{3}$ a $40 \mathrm{mg} \mathrm{L}^{-1} \mathrm{em}$ duas aplicações (aos 45 e 65 dias após o transplante); paclobutrazol (produto comercial Cultar $10 \%$ ) a $100 \mathrm{mg} \mathrm{L}^{-1}$ aos 75 dias após o transplante; $\mathrm{GA}_{3}+$ paclobutrazol aplicados aos 45; 65 e aos 75 dias, respectivamente, após o transplante. Os tratamentos foram aplicados via foliar em ambas as cultivares. As parcelas constaram de 18 plantas, dispostas em três fileiras no espaçamento $40 \times 30 \mathrm{~cm}$, correspondendo a $2,16 \mathrm{~m}^{2}$ de área cultivada.

Para avaliar o efeito dos fitorreguladores sobre o florescimento foi anotado o número de plantas/parcela que apresentavam flores abertas a cada dois dias e, por meio deste levantamento, foi determinado o dia em que cada tratamento, para cada cultivar estudada, atingiu o pleno florescimento, ou seja, quando $50 \%$ das plantas da parcela apresentavam flores abertas.

As colheitas, em número de três por semana, foram de 09/06 a 30/10/99. A cada colheita, os frutos foram classificados em comerciais (peso $>6 \mathrm{~g}$ ), não comerciais (peso $<6 \mathrm{~g}$ ) e danificados/ parcela, os quais foram contados e pesados.

A partir desses dados, foram calculados: produção comercial/planta (PC), obtida a partir da razão entre o peso total dos frutos comerciais por parcela e o número de plantas úteis por parcela; peso médio dos frutos comerciais (PMFC), obtido a partir da razão entre a somatória do peso total dos frutos comerciais por parcela e o número de frutos comerciais; número total de frutos/ planta (NFT), obtido a partir da razão entre a somatória dos frutos produzidos (nas três classes) por parcela e o número de plantas úteis por parcela; porcentagem de frutos comercias/planta $(\% \mathrm{FC})$; porcentagem de frutos não comercias/planta (\%FNC) e, porcentagem de frutos danificados ou mal-formados (\%FD), em relação ao total de frutos de cada parcela.

Os dados de produção foram submetidos à análise de variância, e as médias comparadas pelo teste Duncan no nível de $5 \%$. Para interpretação dos resultados do florescimento, realizou-se a análise de variância, utilizando-se de regressão polinomial para os fatores tratamentos e número de dias. Os dados de porcentagem $(\% \mathrm{FC}, \% \mathrm{FNC} \mathrm{e} \% \mathrm{FD})$ foram transformados em arc sen $\sqrt{ } \mathrm{x} / 100$.

\section{RESULTADOS E DISCUSSÃO}

O pleno florescimento foi atingido nas plantas de 'Seascape', tratadas com água (controle) aos 59 dias, com $\mathrm{GA}_{3}$ aos 54 dias, com paclobutrazol aos 56 dias e com a combinação $\mathrm{GA}_{3}+$ paclobutrazol aos 56 dias. Para a cultivar Oso Grande, o pleno florescimento foi atingido com os mesmos tratamentos, aos 68; 70; 66 e 63 dias, respectivamente.

Considerando que o paclobutrazol foi aplicado aos 75 dias após o transplante das mudas, as plantas tratadas com este produto não tiveram influência do inibidor até a fase de pleno florescimento, igualando-se portanto, àquelas do controle. Da mesma maneira, as plantas do tratamento $\mathrm{GA}_{3}+$ paclobutrazol apresentaram as mesmas condições daquelas tratadas apenas com $\mathrm{GA}_{3}$. Assim, comparando o número de dias para o pleno florescimento nos tratamentos controle e paclobutrazol com aqueles dos tratamentos $\mathrm{GA}_{3}$ e $\mathrm{GA}_{3}+$ paclobutrazol, observou-se que não houve efeito do $\mathrm{GA}_{3}$, e que as pequenas variações ocorridas foram provavelmente devidas ao efeito do ambiente.

Ao longo do período avaliado, a intensidade do florescimento no morangueiro 'Oso Grande' não foi influenciada pelos tratamentos (Figura 1). Esses fatos vêm confirmar a pouca eficiência do paclobutrazol quando aplicado via foliar, visto que pouca ou nenhuma re- 
Tabela 1. Efeito de fitorreguladores na produção comercial por planta (PC); no peso médio dos frutos comerciais (PMFC); no número de frutos totais (NFT), percentual de frutos comerciais $(\% \mathrm{FC})$, percentual de frutos não comerciais $(\% \mathrm{FNC})$ e no percentual de frutos danificados (\%FD), expressos em valores médios/planta, dos morangueiros 'Oso Grande' e 'Seascape'. Caldas, EPAMIG, 1999.

\begin{tabular}{|c|c|c|c|c|c|c|}
\hline Tratamento & PC & PMFC & NFT & $\% \mathrm{FC}^{1}$ & $\% \mathrm{FNC}^{1}$ & $\% F^{1}$ \\
\hline \multicolumn{7}{|c|}{ 'Oso Grande' } \\
\hline Controle & 538,60 & 13,92 & 48,26 & $63,00 b^{*}$ & 19,32 a & $17,88 \mathrm{~b}$ \\
\hline GA3 & 622,44 & 14,47 & 49,69 & $68,00 \mathrm{a}$ & $11,72 b$ & $18,18 b$ \\
\hline Paclobutrazol & 450,05 & 13,64 & 41,98 & $61,95 \mathrm{~b}$ & $13,48 \mathrm{~b}$ & 24,04 a \\
\hline GA3 + Paclobutrazol & 510,66 & 14,30 & 44,87 & $64,90 \mathrm{ab}$ & $13,09 b$ & $20,70 a b$ \\
\hline C.V. $(\%)$ & 20,18 & 6,27 & 12,38 & 4,23 & 19,84 & 11,28 \\
\hline \multicolumn{7}{|c|}{ 'Seascape' } \\
\hline Controle & $261,55 b$ & $12,50 \mathrm{~b}$ & $31,27 \mathrm{~b}$ & $53,89 \mathrm{~b}$ & $23,86 \mathrm{a}$ & $25,23 a b$ \\
\hline $\mathrm{GA}_{3}$ & $471,97 \mathrm{a}$ & 14,85 a & 39,05 a & 64,80 a & $13,33 \mathrm{~b}$ & $20,62 \mathrm{c}$ \\
\hline Paclobutrazol & $249,15 b$ & $13,67 a b$ & $27,95 \mathrm{~b}$ & 53,80 b & 18,64 a & 29,37 a \\
\hline $\mathrm{GA}_{3}+$ Paclobutrazol & 436,17 a & $14,30 \mathrm{a}$ & $39,02 \mathrm{a}$ & 62,19 a & $13,29 b$ & $23,87 \mathrm{bc}$ \\
\hline C.V. $(\%)$ & 17,25 & 6,91 & 12,70 & 4,04 & 17,03 & 10,68 \\
\hline
\end{tabular}

* Médias seguidas de letras diferentes, nas colunas, para cada cultivar, diferem entre si pelo teste Duncan $(\mathrm{P}<0.05)$.

${ }^{1}$ Dados transformados em arc sen vx/100.

serva inibidora fica disponível em tratamentos foliares (Barret e Bartuska, 1982).

A 'Seascape', por ser cultivar de dia neutro, apresentou florescimento mais precoce e em percentuais mais elevados (Figura 2) quando comparada à cv. Oso Grande (Figura 1). Apresentou, ainda, duas fases de máximo florescimento, sendo a primeira entre os 60 e 70 dias e a segunda a partir dos 95 dias. Este comportamento da 'Seascape' é vantajosa para o produtor, uma vez que não há excessiva concentração de produção, o que permite o escalonamento da mãode-obra e da comercialização.

'Seascape' tendeu a responder melhor à aplicação dos tratamentos (Figura 2) que 'Oso Grande'. Ainda, na aplicação do ácido giberélico sozinho ou em combinação com paclobutrazol, houve incremento na intensidade do florescimento ao longo do período avaliado em comparação ao controle. Observou-se também diferença das cultivares na resposta à aplicação do paclobutrazol, sozinho ou em combinação com o GA 3 (Figuras 1 e 2). Quando aplicado sozinho, o paclobutrazol causou inibição mais pronunciada nas plantas da cv. Seascape do que nas da 'Oso Grande’. Já, em combinação com o GA, o efeito inibidor do paclobutrazol foi visível apenas na cultivar Oso Grande. Segundo Castro et al. (1995), esta res-

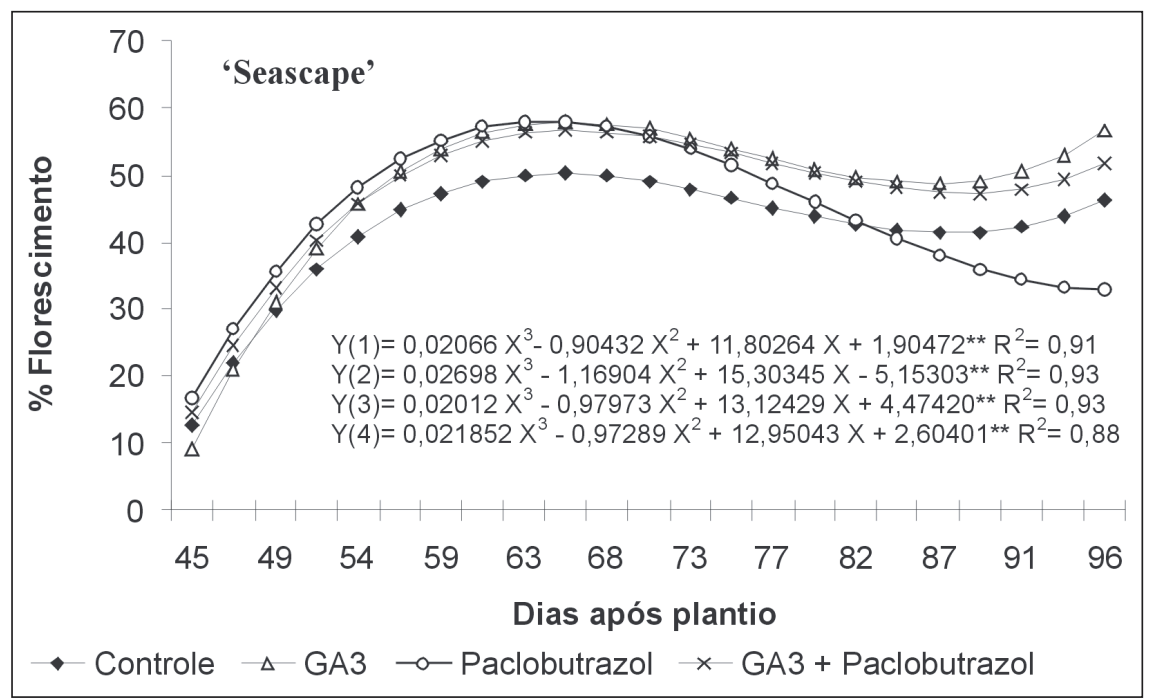

Figura 2. Porcentagem de florescimento, ao longo do período avaliado, do morangueiro cv. Seascape, em função dos diferentes fitorreguladores utilizados. Dados transformados em $\operatorname{arc} \operatorname{sen} \sqrt{\mathrm{X}} / 100$. Caldas, EPAMIG, 1999.

posta pode ser atribuída, principalmente para a 'Seascape', pela ineficiente síntese de giberelina endógena, já que, se esta estivesse ocorrendo satisfatoriamente e exercendo efeitos no desenvolvimento, seria inibida pelo paclobutrazol, inibidor da biossíntese do ácido giberélico. Este fato pode ser melhor visualizado nas plantas de 'Seascape' tratadas com $\mathrm{GA}_{3}+$ paclobutrazol, com suplementação de $\mathrm{GA}_{3}$ antes do tratamento com paclobutrazol, resultando em efeito menos pronunciado, quando comparado com plantas tratadas unicamente com paclobutrazol.

Verificou-se que, para 'Oso Grande', com exceção dos parâmetros \% \%C, \% $\mathrm{FNC}$ e $\% \mathrm{FD}$, em que foram observadas diferenças significativas a $5 \%$ de probabilidade, os demais não foram alterados em função dos tratamentos com fitorreguladores (Tabela 1). Já para as plantas da cv. Seascape ocorreu resposta positiva para todos os parâmetros avaliados, em função dos tratamentos que 
continham $\mathrm{GA}_{3}$. A hipótese de Castro et al (1995), relatada anteriormente, da mesma forma que para o florescimento, explica perfeitamente esta resposta diferenciada entre as cultivares.

O aumento da produção de frutos comerciais/planta (PC) e do peso médio dos frutos comerciais (PMFC), como resultado da aplicação de $\mathrm{GA}_{3}$, foi também verificado por López-Galarza et al. (1989), Miranda-Stalder et al. (1990) e por Castro et al. (1995).

Observou-se, também, que a porcentagem de frutos danificados ou mal-formados variou conforme o tratamento e a cultivar. A aplicação de $\mathrm{GA}_{3}$ causou redução neste parâmetro somente nas plantas da cv. Seascape, enquanto que o paclobutrazol sozinho causou incremento somente em 'Oso Grande', diferindo estatisticamente do controle. Esta redução na produção de frutos desta classe é atribuída principalmente à mudança na arquitetura da planta, causada pelo $\mathrm{GA}_{3}$, que é um promotor do desenvolvimento vegetativo, passando com isto a apresentar folhagem mais aberta e menos adensada. Isto possibilitou maior penetração da luz solar no perfil da planta, permitindo o aumento na taxa fotossintética, e redução na umidade relativa, restringindo em muito a proliferação de doenças e pragas limitantes do desenvolvimento do morangueiro. Por outro lado o paclobutrazol isolado causou o inverso, ou seja, maior adensamento, devido à inibição do desenvolvimento do pecíolo das plantas.

O acréscimo na produção comercial (PC) verificado na cv. Seascape devese ao incremento do peso médio dos frutos comerciais e da porcentagem de frutos comerciais $(\% \mathrm{FC})$, resultante do efeito do $\mathrm{GA}_{3}$ exógeno aplicado no crescimento do fruto do morangueiro. $\mathrm{O}$ $\mathrm{GA}_{3}$ atua na elevação da taxa fotossintética, aumentando a produção de carboidratos necessários para atender a expansão celular destes frutos. $\mathrm{O}$ volume do fruto é devido tanto ao número quanto ao tamanho das células, por estas serem constituídas principalmente de protoplasma (Chitarra e Chitarra, 1990). Com o crescimento aparecem os vacúolos, onde ocorre acúmulo de carboidratos e de outros compostos translocados das folhas. O efeito do $\mathrm{GA}_{3}$, no acréscimo da produção comercial da cv. Seascape pode também estar relacionado ao aumento na diferenciação floral (Figura 2).

Os resultados obtidos evidenciaram que a resposta à aplicação dos fitorreguladores $\mathrm{Ga}_{3}$ e paclobutrazol em morangueiro depende da cultivar, como foi o caso da cv. Seascape, de dia neutro, que respondeu melhor à aplicação de GA 3 que a 'Oso Grande', de dia curto. Uma outra constatação foi que o paclobutrazol, quando aplicado via foliar, aos 75 dias após o transplante, reduziu o florescimento. Entretanto, há necessidade de mais pesquisas sobre dosagens e épocas de aplicação do paclobutrazol no morangueiro.

\section{LITERATURA CITADA}

BARRETT, J.E.; BARTUSKA, C.A. PP333 effects on stem elongation dependent on site of application. HortScience, v.17, n.5, p.737, 1982. CASTRO, P.R.C.; COLLETTI JUNIOR, R.; MINAMI, K.; DEMÉTRIO, C.G.B.; PIEDADE, S.M.S. Frutificação do morangueiro cultivar Campinas sob efeito de reguladores vegetais. Revista de Agricultura, Piracicaba, v.70, p.277-289, 1995.
CHITARRA, M.I.F.; CHITARRA, A.B. Pós colheita de frutos e hortaliças: fisiologia e manuseio. Lavras: ESAL/FAEPE, 1990. 320 p.

DEYTON, D.E.; SAMS, C.E.; CUMMINS, J.C. Strawberry growth and photosynthetic responses to paclobutrazol. HortScience, v.26, n.9, p.11781180, 1991.

DUARTE FILHO, J.; CUNHA, R.J.P.; ALVARENGA, D.A.; PEREIRA, G.E.; ANTUNES, L.E.C. Aspectos do florescimento e técnicas empregadas objetivando a produção precoce em morangueiro. Informe Agropecuário, Belo Horizonte, v.20, n.198, p.30-35, 1999.

LÓPEZ-GALARZA， S.; PASCUAL， B.; ALAGARDA, J.; MAROTO, J.V. The influence of winter gibberellic acid applications on earliness, productivity and other parameters of quality in strawberry cultivation (Fragaria X ananassa Duch.) on the spanish mediterranean coast. Acta Horticulturae, n.265, p.217-222, 1989.

LUCCHESI, A.A.; MINAMI, K. Utilização de fito-reguladores de crescimento em morangueiro (Fragaria spp.): influência no ciclo da cultura e na produção final. Anais da Escola Superior de Agricultura "Luiz de Queiroz", Piracicaba, v.37, p.485-515, 1980.

MIRANDA-STALDER, S.H.G.; APPEZZATODA GLÓRIA, B.; CASTRO, P.R.C. Efeitos de reguladores de crescimento nas características organográficas e na produtividade do morangueiro 'Sequóia'. Anais da escola Superior de Agricultura "Luiz de Queiroz", Piracicaba, v.47, p.317-344, 1990.

NISHIZAWA, T. The effect of paclobutrazol on growth and yield during first year greenhouse strawberry production. Acta Horticulturae, n.329, p.51-53, 1993.

RAMINA, A.; TONUTTI, P.; TOSI, T. The effect of paclobutrazol on strawberry growth and fruiting. Journal of Hoticultural Science, v.60, n.4, p.501-506, 1985.

ROUDEILLAC, P. Situation de la production de fraises dans le monde: perspectives en Europe. In: DUARTE FILHO, J.; CANÇADO, G.M.A.; REGINA, M.A.; ANTUNES, L.E.C.; FADINI, M.A.M., eds. Morango: tecnologia de produção e processamento. Caldas: EPAMIG, 1999. p.1-38. 